\title{
Computer-Aid Molecular Docking Technology in Cereal Mycotoxin Analysis
}

\author{
Jinying Chen ${ }^{1}$, Jin $\mathrm{Ye}^{2}$, Yan Zhang ${ }^{3}$, Chen Shuai ${ }^{1}$ and Qiang Yuan ${ }^{3}$ \\ 1. Sino Grain Chengdu Storage Research Institute Co. Ltd., Chengdu 610000, China \\ 2. Academy of National Food and Strategic Reserves Administration, No. 11 Baiwanzhuang Str, Xicheng District, Beijing 100037, \\ China
}

3. Standards and Quality Center of National Food and Strategic Reserves Administration, Beijing 100037, China

\begin{abstract}
Computer-aid molecular docking is a simulative process that receptors and ligands recognize each other through energy matching and geometric matching. It is widely used in bioactive compounds simulative screening and preliminary exploring the bioactivity and toxicity of molecular, which plays an important guiding role in toxicity and bioactivity study of molecular entities. In our study, we used the computer-aid molecular docking software-discovery studio 3.1 client to test the mechanism of aflatoxins such as aflatoxin B1, B2, M1, M2, G1, G2 and the results of our experiment help to illustrate the pathway of aflatoxin's toxication. We also used this technology to test the preliminary toxicity of zearalenone (ZEN) and its two degradation products: $\alpha$-zearalenol $(\alpha-Z O L)$ and $\beta$-zearalenol ( $\beta$-ZOL), which indicates that these three products possessed significant estrogenic activity. The order of the estrogenic activity is: $\alpha$-zearalenol $>$ zearalenone $>\beta$-zearalenol.
\end{abstract}

Key words: Computer-aid molecular docking, aflatoxin, zearalenone, toxicity.

\section{Introduction}

Over the last few decades, computer-aid molecular docking technology has grown significantly in the development of new drug molecules. As a powerful technique, it relives the tension in drug discovery such as time-consuming, high-cost and low success rates. Additionally, with rapid development of biological structures and computer technology, this technology is widely used in mycotoxin toxicity pathway research $[1,2]$.

Using direct docking methods or virtual high-throughput screening (HTS), affinity of molecules to targets can be estimated based on compounds' conformation and complementarity with residues in binding site. Through analysis of binding free energies, further filtering and optimization of possible molecules subsequently follow, a limited number of lead molecules are selected for in vitro

Corresponding author: Jinying Chen, Ph.D., associate professor, research field: biochemistry. bioactivity tests. Automatic docking is aimed at the determination of the optimal position and orientation of molecule in binding pocket of particular protein target $[3,4]$. Quality of protein-ligand interactions is usually qualified by ligand efficiency (LE) and average binding energy per non-hydrogen atom of the ligand. While virtual high-throughput screening is performed to evaluate libraries of molecules for binding affinity to the protein target, this HTS strategy can shortlist compounds that are most likely to bind to the selected target with the highest affinity. A plethora of software has been developed for molecular docking including Dock, GOLD, and AutoDock, etc. In addition, other docking strategies such as flexible ligand docking, fragment docking and fragment growing have been used in high-throughput docking campaigns $[5,6]$.

In our research, we used the computer-aid molecular docking software-discovery studio 3.1 client to test the mechanism of aflatoxins and illustrate the pathway of aflatoxin's toxication. We also used 
this technology to test the preliminary toxicity of zearalenone and its two degradation products: $\alpha$-zearalenol and $\beta$-zearalenol, which indicates that these three products possessed significant estrogenic activity.

\section{Materials and Methods}

The 2D structure of aflatoxin B1 and oltipraz was generated by ChemDraw Ultra 12.0 (Cambridge Soft, Cambridge, MA, USA). A homology model of protein receptor was constructed from crystal structure of PDB: obtained from the RCSB protein Data Bank.

Water molecules were removed and $\mathrm{H}$ atoms were added to the structure; 3D structures of the compounds were generated and optimized by the Discovery Studio 2.1 package (Accelrys, San Diego, CA, USA). The receptor-grid files were carried out using a grid-receptor generation program using default settings after ensuring that the ligands and the protein are in correct form. The GOLD program in the Discovery Studio software was used to perform the docking simulations, which allows full flexibility of the ligand.

The structures of the aflatoxins, zearalenone, $\alpha$-zearalenol and $\beta$-zearalenol were drawn in Chem3D with standard lengths and angles. The Gasteiger-Huckel charge, with a distance-dependent dielectric function, and AM1 docking calculations were applied for the minimization of the molecules. To modify the structure of receptor, missing atoms, bonds, and contacts were checked, hydrogen atoms were added to the enzyme structure, and water molecules were removed. Intercalation models were optimized using the Chemistry at HARvard Macromolecular Mechanics (CHARMM) force field with the added parameters. After performing the docking simulation, the scores of the docked conformers were ranked and the best binding modes in the cavity were picked out.

\section{Results and Discussion}

\subsection{Aflatoxin Toxicity Analysis}

Aflatoxins are among the most potent natural hepato-carcinogenic products, which are produced mainly by the fungi Aspergillus flavus and $A$. parasiticus. Nowadays scientist has separated and identified twelve aflatoxins analogues including aflatoxin B1, B2, G1, M1, P1, Q1, H1, GM, B2a and aflatoxicol (Fig. 1). The basic structures of aflatoxins are dihydrofuran and coumarin and aflatoxin B1 (AFTB1), which is the analogue of dihydrofuran oxynaphthalene, and contains two furan ring (the basic toxic structure) and one cumarin $[7,8]$.

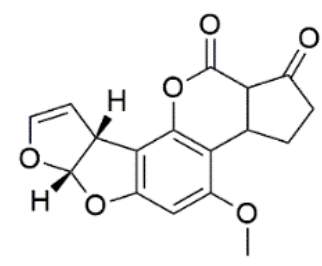

Aflatoxin B2

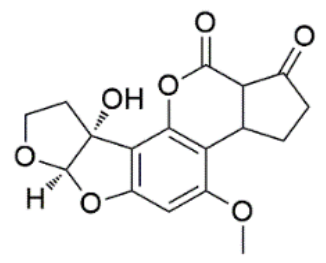

Aflatoxin M2

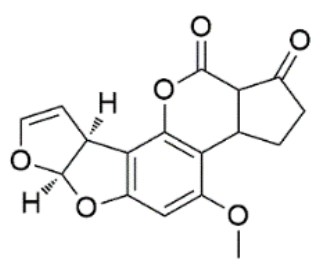

Aflatxoin B1<smiles>COc1cc2c(c3c1C(O)C1C(=O)OCCC31)OC(=O)CO2</smiles>

Aflatoxin G1<smiles>COc1cc2c(c3c1C1CCC(=O)C1C(=O)O3)[C@@]1(O)C=CO[C@@H]1O2</smiles>

Aflatoxin M1<smiles>COc1cc2c3c(C4CCOC(=O)C4)c1C(CO2)C(=O)O3</smiles>

Aflatoxin G2

Fig. 1 The chemical structures of aflatoxins metabolic products. 
3.1.1 The Interaction between AFTB1 and Receptor Proteins

In our research, we chose some kinds of proteins which play significant roles in cell apoptosis, estrogen metabolism, immunosuppression and digestive system function as the potential targets of the toxic pathway of aflatoxin B1, including Caspase-1, cell division protein kinase 2, serine/threonine protein kinase chk1, progesterone receptor, androgen receptor, estrogen receptor, alpha-thrombin, prostaglandin $\mathrm{g} / \mathrm{h}$ synthase 2 , estradiol 17-beta-dehydrogenase 1, macrophage migration inhibitory factor and estrogen sulfotransferase. We made AFTB1 molecular docked with the above proteins by molecular docking software, the results were shown in Table 1. As the results showed, estrogen sulfotransferase was proved to be the best dock receptor of AFTB1 and the scores were 130.22 and -10.9013 by Libdock and CDocker, respectively.

3.1.2 The Interaction between AFTB1 and Estrogen Sulfotransferase

We made AFTB1 docked with estrogen sulfotransferase, and the docking result was shown in Fig. 2. AFTB1 could perfectly dock into the formed cavity of estrogen sulfotransferase protein and there was formed cation- $\pi$ interaction between the benzene ring and arginine residue (ARG B:129). The carbonyl group in coumarin formed hydrophobic interactions with tyrosine residue (TYR B:192) and the oxygen atom in furan ring formed hydrophobic interactions with arginine residue (ARG B:256).

3.1.3 The Interaction between AFTB2 and Estrogen Sulfotransferase

We made AFTB2 docked with estrogen sulfotransferase, and the docking result was shown in Fig. 3. AFTB2 could perfectly dock into the formed cavity of estrogen sulfotransferase protein and there was formed cation- $\pi$ interaction between the benzene ring and arginine residue (ARG B:129). The carbonyl group in coumarin formed hydrophobic interactions with arginine residue (ARG B:256), allysine residue (LYS B:257) and glycine residue (GLY B:258).

3.1.4 The Interaction between AFTB M1 and Estrogen Sulfotransferase

We made AFTB M1 docked with estrogen sulfotransferase, and the docking result was shown in Fig. 4. AFTB M1 could perfectly dock into the formed cavity of estrogen sulfotransferase protein and there was formed cation- $\pi$ interaction between the benzene ring and arginine residue (ARG B:129). The phenolic hydroxy group in furan ring formed hydrophobic interactions with arginine residue (ARG B:256), allysine residue (LYS B:257) and glycine residue (GLY B:258). The oxygen atom in furan ring formed hydrophobic interactions with arginine residue (ARG B:256).

Table 1 AFTB1 targets predicted by Libdock and CDocker.

\begin{tabular}{llll}
\hline Protein & PDB & Libdock score & CDocker socre \\
\hline Caspase-1 & 3D6F & 82.8002 & $/$ \\
Cell division protein kinase 2 & 1HCK & 97.2643 & -0.549952 \\
Serine/threonine-protein kinase chk1 & 1IA8 & 100.060 & $/$ \\
Progesterone receptor & $1 \mathrm{~A} 28$ & 98.6966 & $/$ \\
Androgen receptor & 5DIU & 113.528 & -20.9024 \\
Estrogen receptor & 1QKM & 110.000 & -22.1885 \\
Alpha-thrombin & 1ABI & 82.2093 & $/$ \\
Prostaglandin g/h synthase 2 & 1PD2 & 117.653 & $/$ \\
Estradiol 17-beta-dehydrogenase 1 & 1BHS & 98.6731 & -968.253 \\
Macrophage migration inhibitory factor & 1MFI & 90.8723 & -10.9013 \\
Estrogen sulfotransferase & 1HY3 & 130.022 & -16.2633 \\
Sex hormone-binding globulin & 1D2S & 111.558 & \\
\hline
\end{tabular}



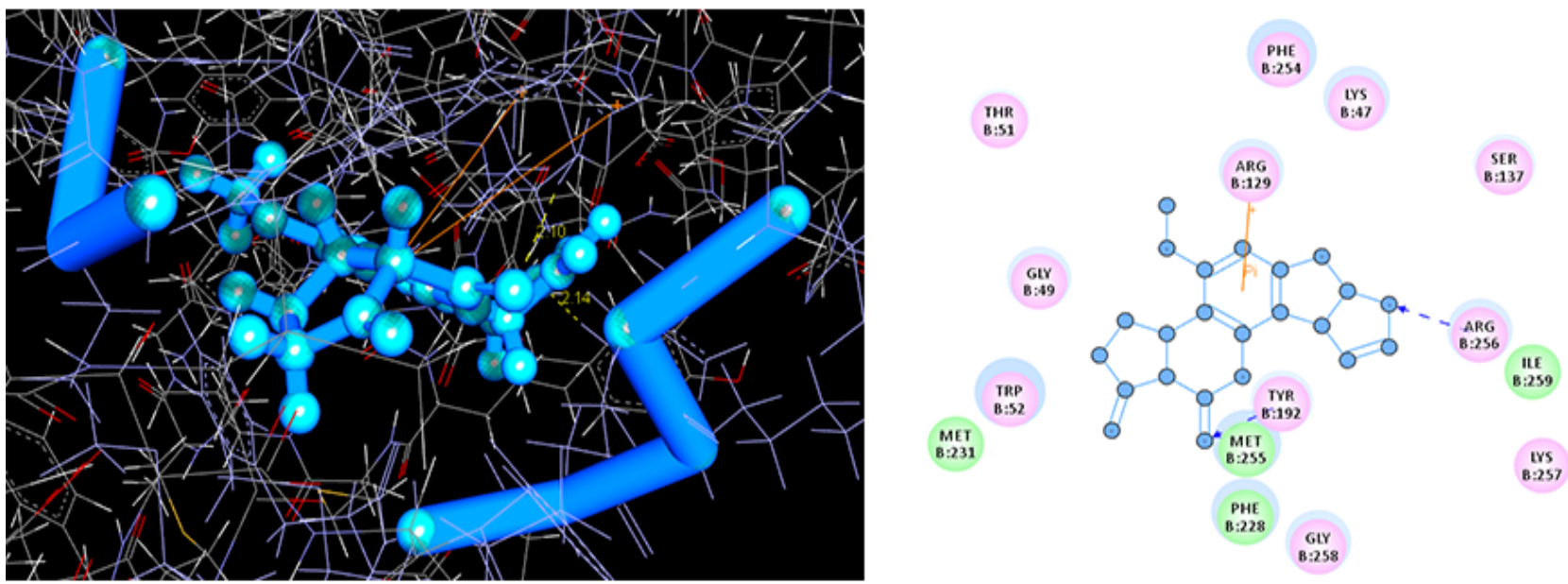

Fig. 2 The interaction between AFTB1 and estrogen sulfotransferase.
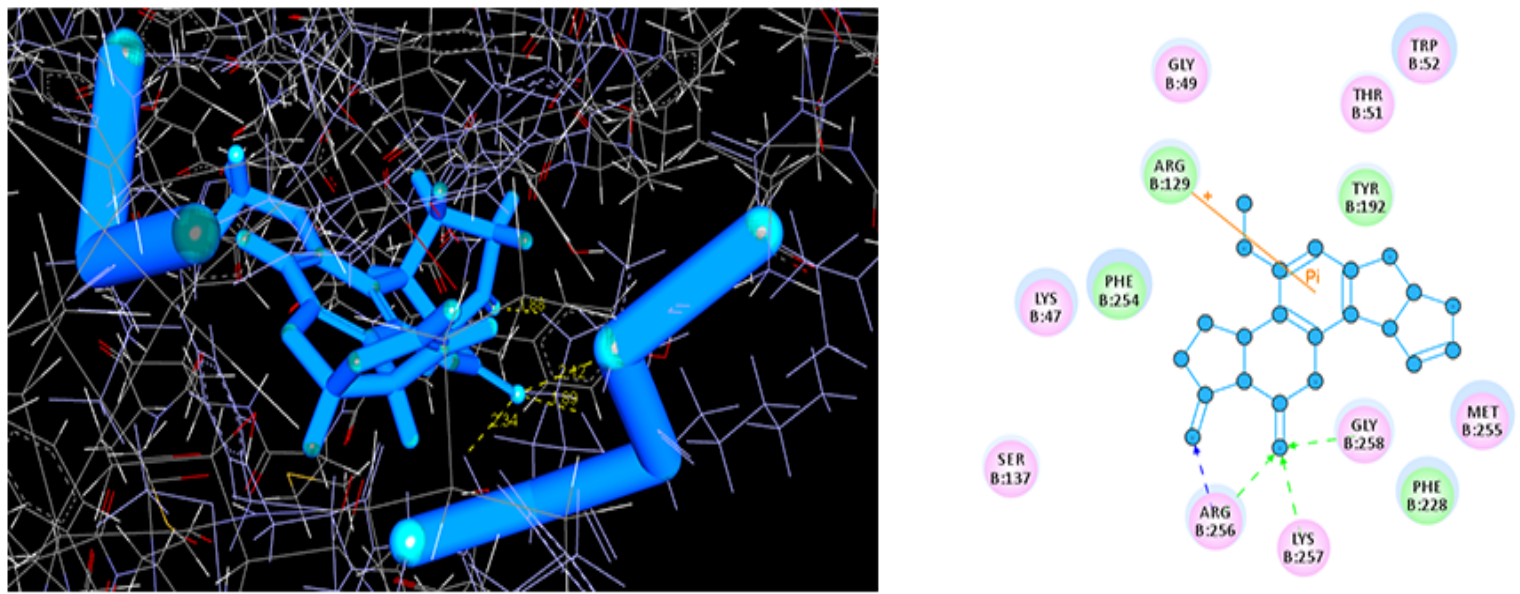

Fig. 3 The interaction between AFTB2 and estrogen sulfotransferase.
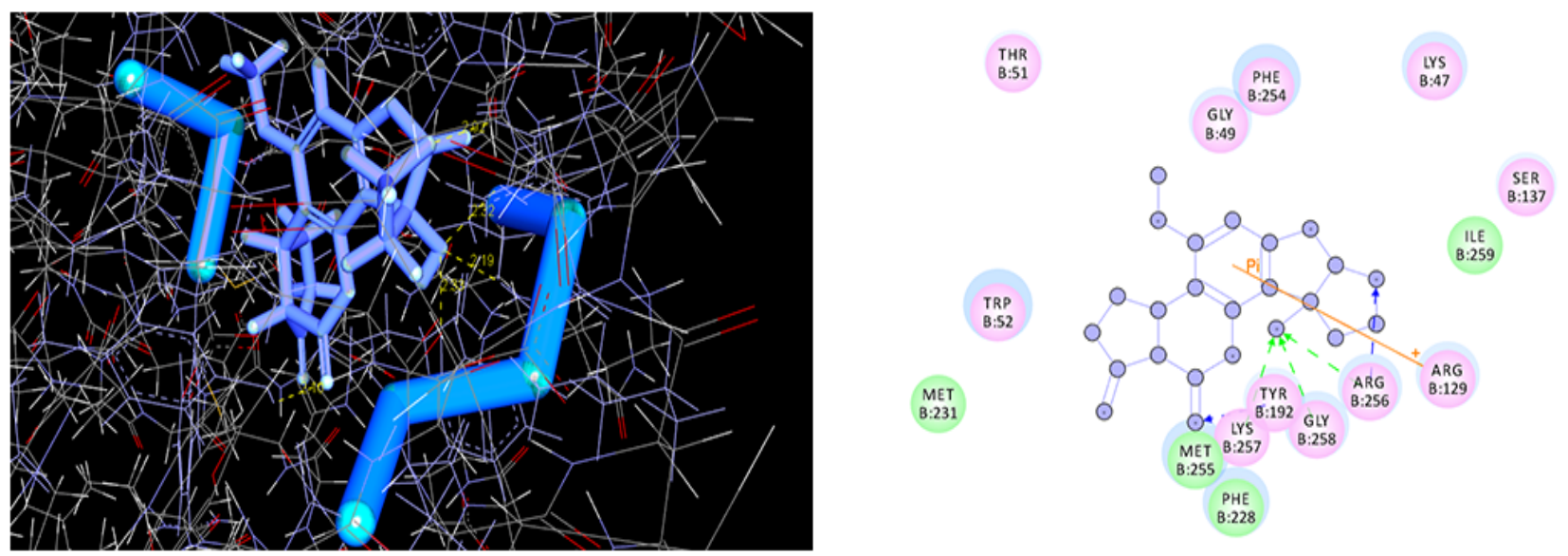

Fig. 4 The interaction between AFTB M1 and estrogen sulfotransferase.

3.1.5 The Interaction between AFTB M2 and Estrogen Sulfotransferase

We made AFTB M2 docked with estrogen sulfotransferase, and the docking result was shown in
Fig. 5. There was formed cation- $\pi$ interaction between the benzene ring and arginine residue (ARG B:129). The phenolic hydroxy group in furan ring formed hydrophobic interactions with arginine residue (ARG 

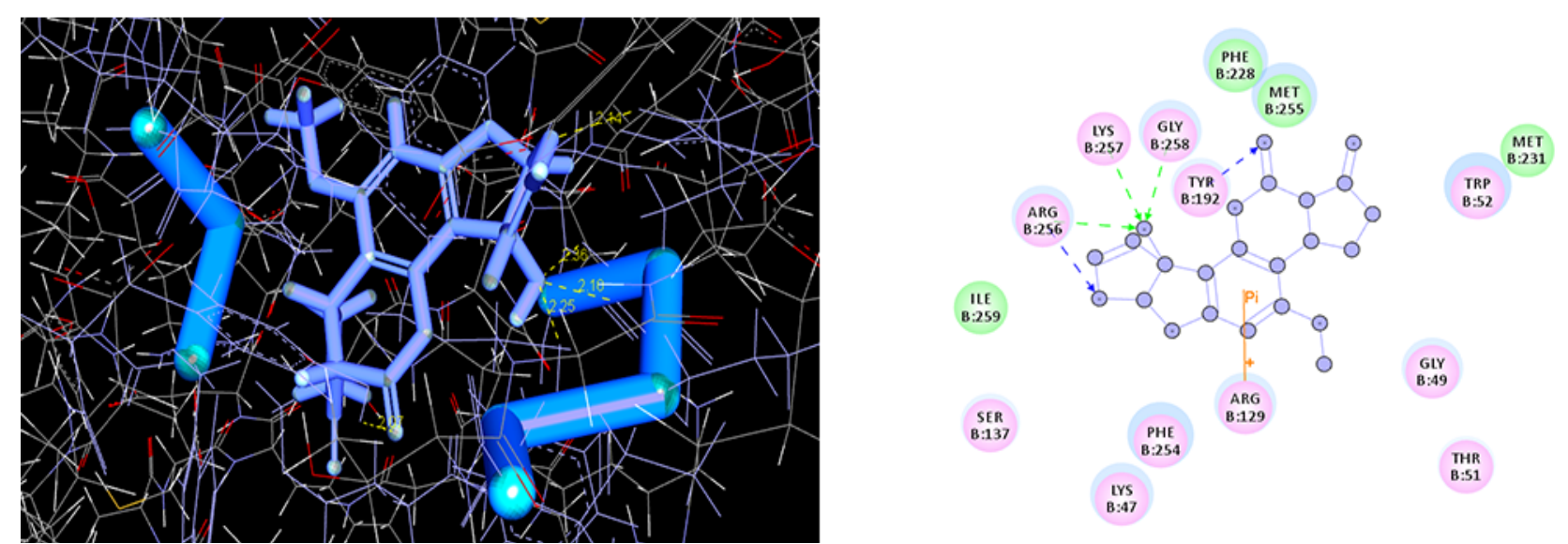

Fig. 5 The interaction between AFTB M2 and estrogen sulfotransferase.
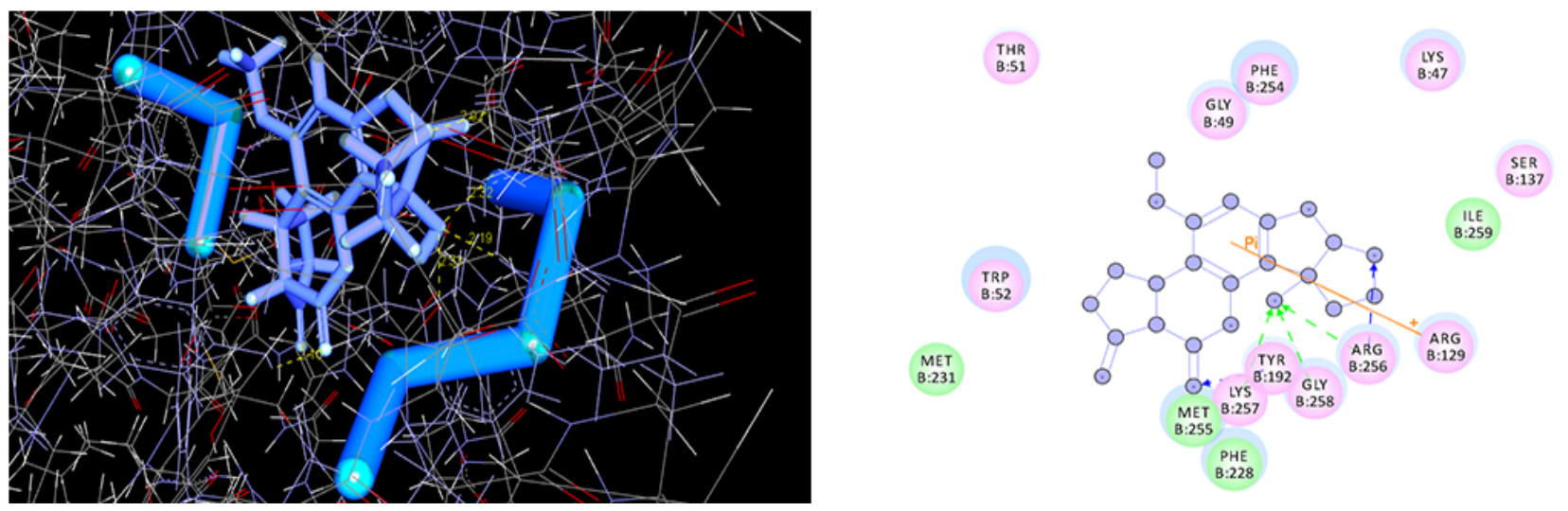

Fig. 6 The interaction between AFTB G1 and estrogen sulfotransferase.

B:256), lyrosine residue (TYR B:192) and glycine residue (GLY B:258). The oxygen atom in furan ring formed hydrophobic interactions with arginine residue (ARG B:256), the carbonyl group in coumarin formed hydrophobic interactions with tyrosine residue (TYR B:192).

3.1.6 The Interaction between AFTB G1 and Estrogen Sulfotransferase

We made AFTB G1 docked with estrogen sulfotransferase, and the docking result was shown in Fig. 6 . There was formed cation- $\pi$ interaction between the benzene ring and arginine residue (ARG B:129). The inside carbonyl group in coumarin formed hydrophobic interactions with arginine residue (ARG B:256), allysine residue (LYS B:257) and glycine residue (GLY B:258). The outside carbonyl group in coumarin formed hydrophobic interactions withallysine residue (LYS B:47). The oxygen atom in furan ring formed hydrophobic interactions with arginine residue (ARG B:256).

3.1.7 The Interaction between AFTB G2 and Estrogen Sulfotransferase

We made AFTB G2 docked with estrogen sulfotransferase, and the docking result was shown in Fig. 7. There was no formed cation- $\pi$ interaction between the benzene ring and any residue. The inside carbonyl group in coumarin formed hydrophobic interactions with arginine residue (ARG B:256), allysine residue (LYS B:257) and glycine residue (GLY B:258). The outside carbonyl group in coumarin formed hydrophobic interactions withallysine residue (LYS B:47).

3.1.8 The Binding Energy and Docking Score between Ligand and Receptors

The results of binding energy and docking score between aflatoxin B1, B2, M1, M2, G1, G2 and estrogen sulfotransferase were shown in Table 2. The 

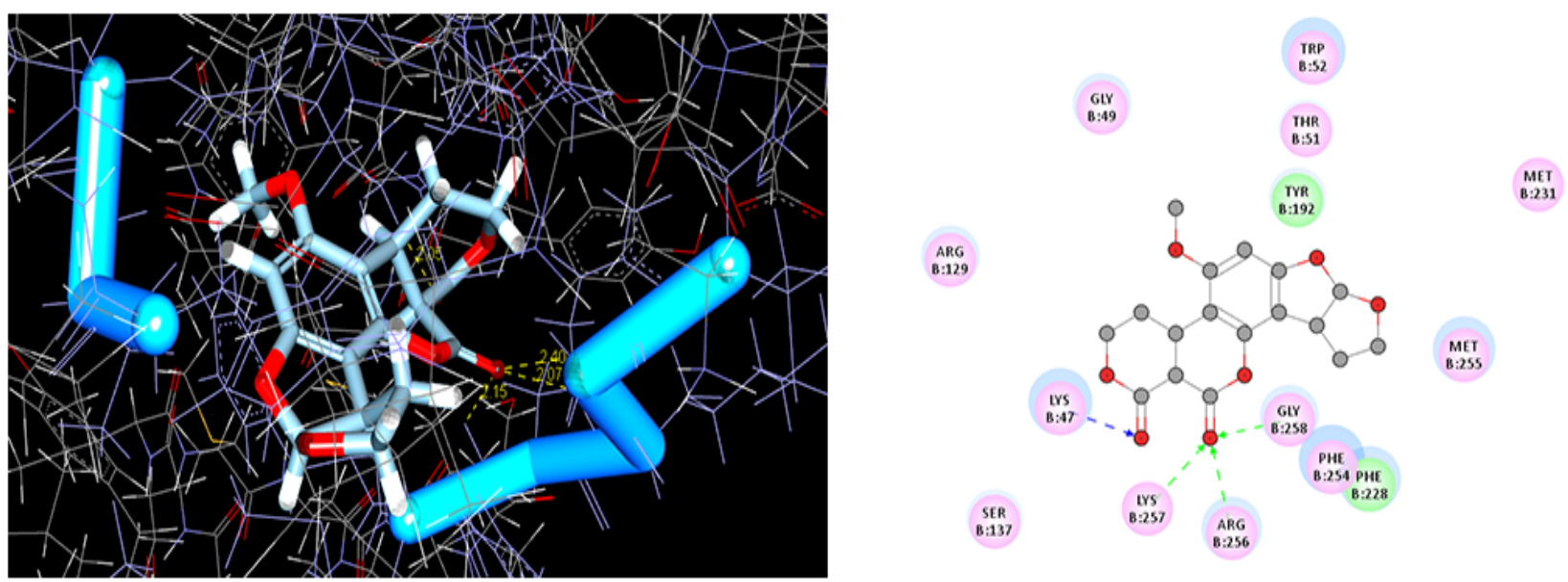

Fig. 7 The interaction between AFTB G2 and estrogen sulfotransferase.

Table 2 The binding energy and docking score between ligand and receptors.

\begin{tabular}{lll}
\hline Ligand & Binding energy & Number \\
\hline Aflatoxin M2 & -8.21584 & 10 \\
Aflatoxin M1 & -8.5654 & 10 \\
Aflatoxin B1 & -10.9013 & 10 \\
Aflatoxin G2 & -14.1769 & 10 \\
Aflatoxin G1 & -19.5298 & 10 \\
Aflatoxin B2 & -20.2356 & 10 \\
\hline
\end{tabular}

binding energy between AFTB1, AFTB2, AFTB M1, AFTB M2, AFTB G1, AFTB G2 with estrogen sulfotransferase was $-10.9013 \mathrm{kcal} / \mathrm{mol},-20.2356$ $\mathrm{kcal} / \mathrm{mol}, \quad-8.5654 \mathrm{kcal} / \mathrm{mol}, \quad-8.2156 \mathrm{kcal} / \mathrm{mol}$, $-19.5298 \mathrm{kcal} / \mathrm{mol},-14.1768 \mathrm{kcal} / \mathrm{mol}$. AFTB M1 was proved to be the best binding ligand with estrogen sulfotransferase receptor.

\subsection{Estrogenic Effect in Zearalenone}

Zearalenone (6-(10-hydroxy-6-oxo-trans-1-undece nyl)-resorcylic acid lactone, abbreviated as ZEN) is a is produced by Fusarium roseum and is usually isolated from moldy corn [9]. Zeranol, a synthetic tetrahydro-derivative of $\mathrm{ZEN}$, has been used as a growth promoter for food-producing animals [10]. Earlier studies have shown that ZEN and ZOL have strong estrogenic effects, and each of them was reported to have a similar dose-response curve pattern in stimulating uterine weight gains in neonatal rats or immature mice [11]. The most common ZOLs are $\alpha-Z O L$ and $\beta-Z O L$, which are shown in Fig. 8 .
3.2.1 The Interaction between ZEN and $\alpha$-estrogen Receptor Protein

In order to prove the estrogen effect of $\mathrm{ZEN}$ in molecular stage, we used the molecular docking software to simulate the binding situation between ZEN and $\alpha$-estrogen receptor protein, as Fig. 9 showed, ZEN can perfectly dock into the cavity of estrogen receptor protein crystal structure, and the hydroxy group of benzene ring formed strong hydrophobic interactions with contiguous alanine residue (ALA350) and glutamic acid residue (GLU353), which strengthen the binding ability between the ligands and the receptors.

3.2.2 The Interaction between ZEN and $\beta$-estrogen Receptor Protein

We used the molecular docking software to simulate the binding situation between ZEN and $\beta$-estrogen receptor protein, as Fig. 10 showed, the 3-hydroxy group in benzene ring of ZEN formed strong hydrophobic interactions with contiguous histidine residue (HIS475) and glycine residue (GLY472), 
which strengthen the binding ability between the ligands and the receptors.

3.2.3 The Interaction between ZOL and Estrogen Receptor Protein

We have proved that ZEN molecule had favorable binding ability with $\alpha$-estrogen receptor protein and $\beta$-estrogen receptor protein, which demonstrated that ZEN possessed strong estrogen effect. In the following study, we would prove whether ZOL had the similar estrogen effect.

We used the molecular docking software to simulate the binding situation between $\alpha$-ZOL, $\beta$-ZOL and $\alpha$-estrogen receptor protein, $\beta$-estrogen receptor protein. From Fig. 11, it was proved that $\alpha-Z O L$ can perfectly dock into the cavity of estrogen receptor protein crystal structure, 3-hydroxy group in benzene ring of $\alpha$-ZOL formed strong hydrophobic interactions with contiguous arginine residue (ARG394) in $\alpha$-estrogen receptor protein and glycine residue (GLY472) in $\beta$-estrogen receptor protein; aliphatic hydroxyl group in aliphatic chain formed strong<smiles>[Z][C@H](C)/C=C/CCCC(=O)CCCC[C@H](C)OC(=O)c1ccc(O)cc1O</smiles><smiles>C[C@@H]1CCC[C@H](O)CCC/C=C/c2cc(O)cc(O)c2C(=O)O1</smiles>

$\alpha$-zearalenol<smiles>C[C@@H]1CCC[C@H](O)CCC/C=C/c2cc(O)cc(O)c2C(=O)O1</smiles>

$\beta$-zearalenol<smiles>C[C@]12CC[C@@H]3c4ccc(O)cc4CC[C@H]3[C@@H]1CC[C@@H]2O</smiles>

17 $\beta$ - estradiol

Fig. 8 Chemical structures of ZEN, $\alpha$-ZOL, $\beta$-ZOL and $\beta$-estriol.
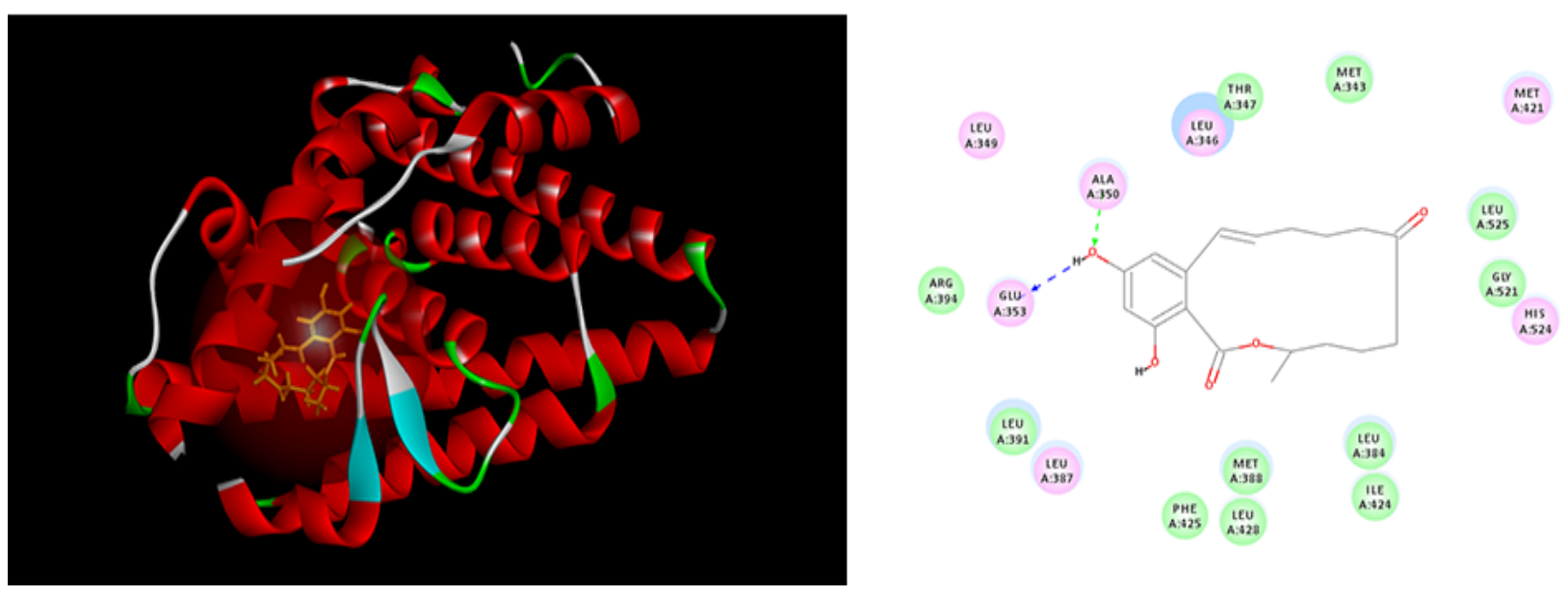
Fig. 9 The interaction between ZEN and $\alpha$-estrogen receptor protein.
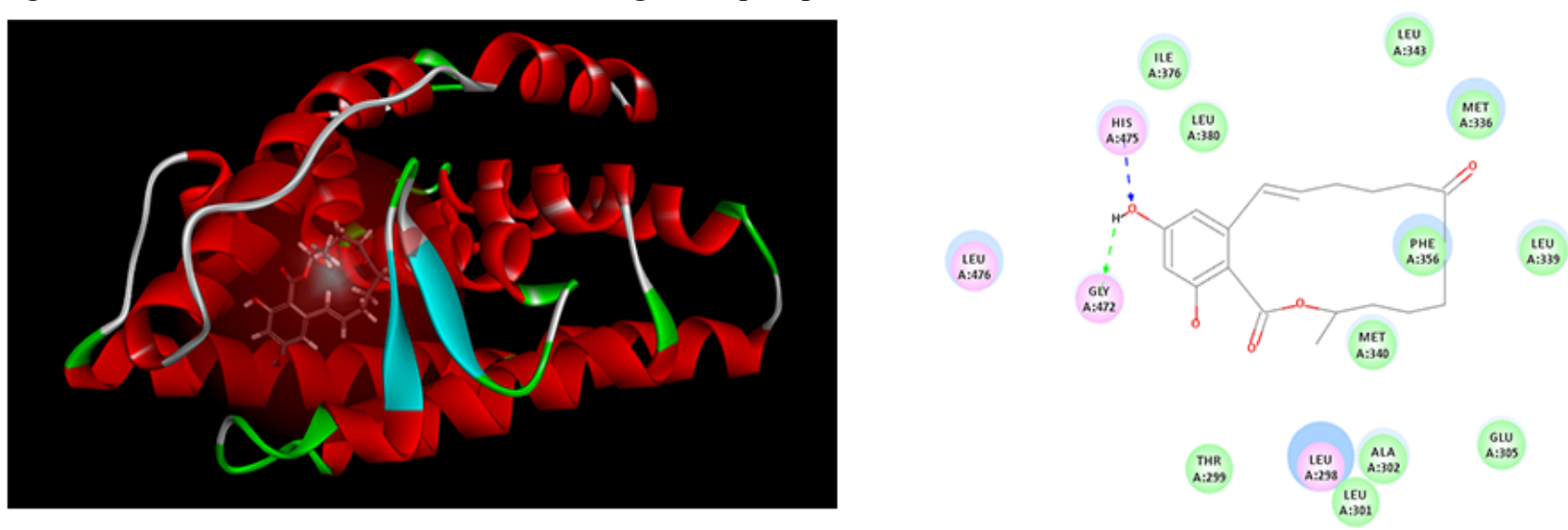

Fig. 10 The interaction between ZEN and $\beta$-estrogen receptor protein.
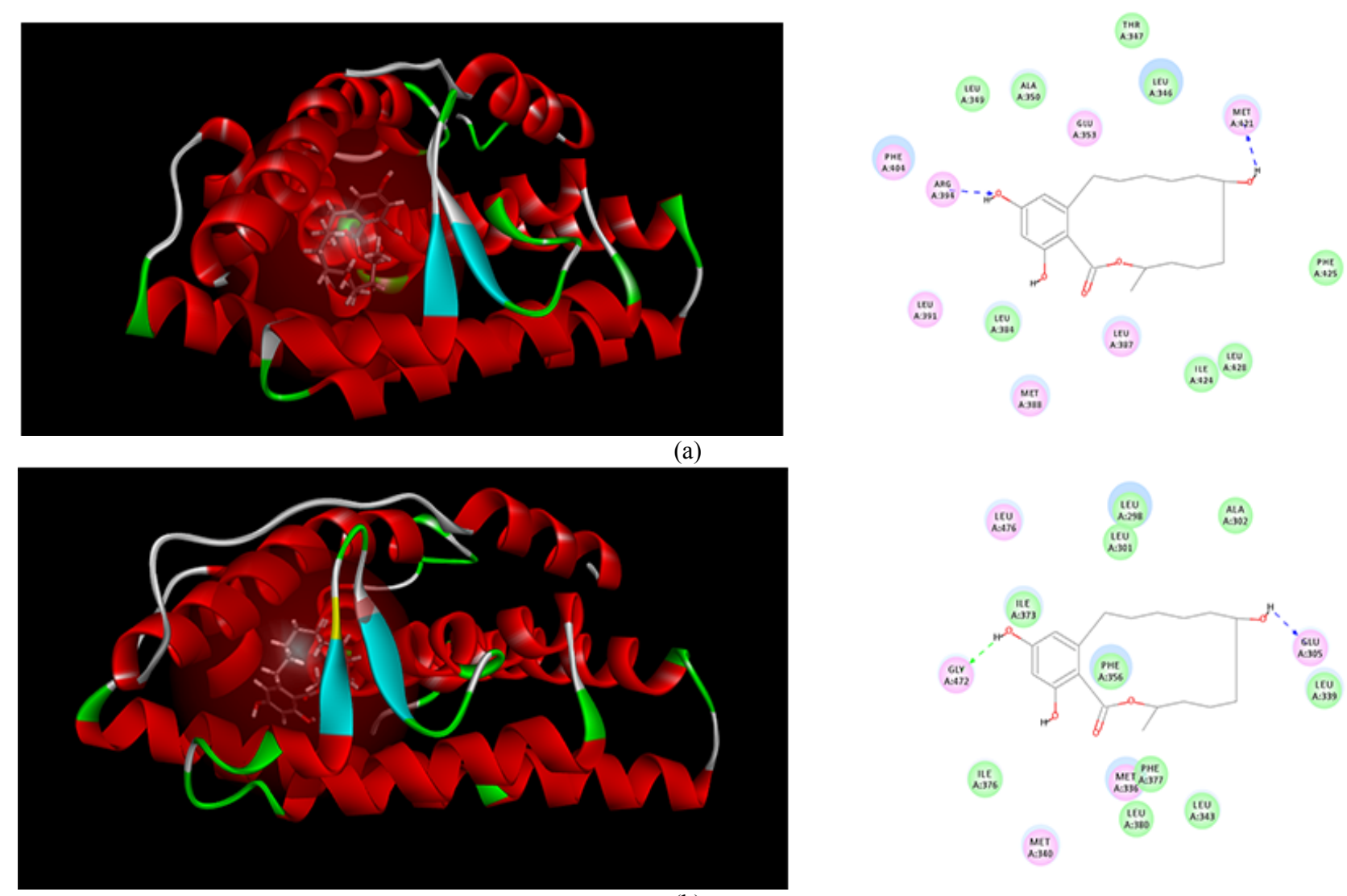

Fig. 11 The interaction between $\alpha-\mathrm{ZOL}$ and estrogen receptor protein.

hydrophobic interactions with methionine (MET421) in $\alpha$-estrogen receptor protein and glutamic residue (GLU305) in $\beta$-estrogen receptor protein. The binding ability between $\beta$-ZOL and estrogen receptor protein was relatively weak and there only one existed hydrophobic interaction according to the docking results (Fig. 12).
3.2.4 The Binding Ability and Docking Score between Ligands and Recepor

The results of binding energy and docking score between 17 $\beta$-estradiol (positive control), ZEN, $\alpha-Z O L$, $\beta-Z O L$ and estrogen receptor protein were shown in Table 3. The order of the estrogenic activity is: 
$\alpha$-zearalenol $>$ zearalenone $>\beta$-zearalenol.

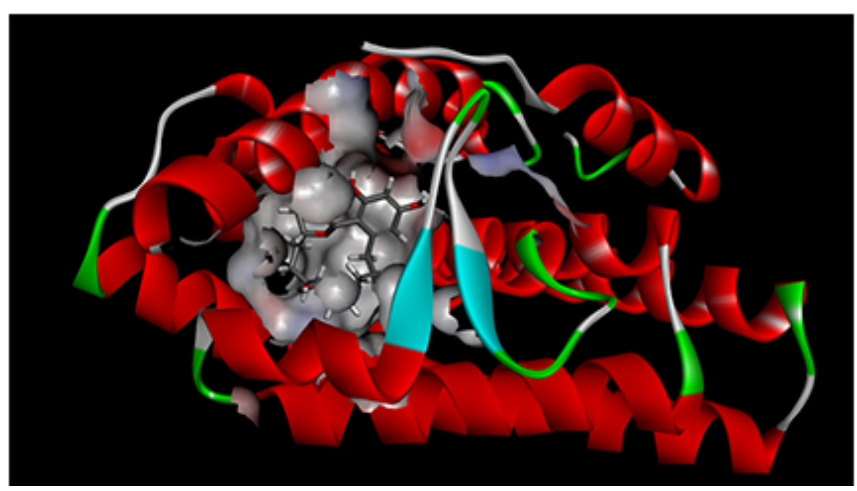

(a)

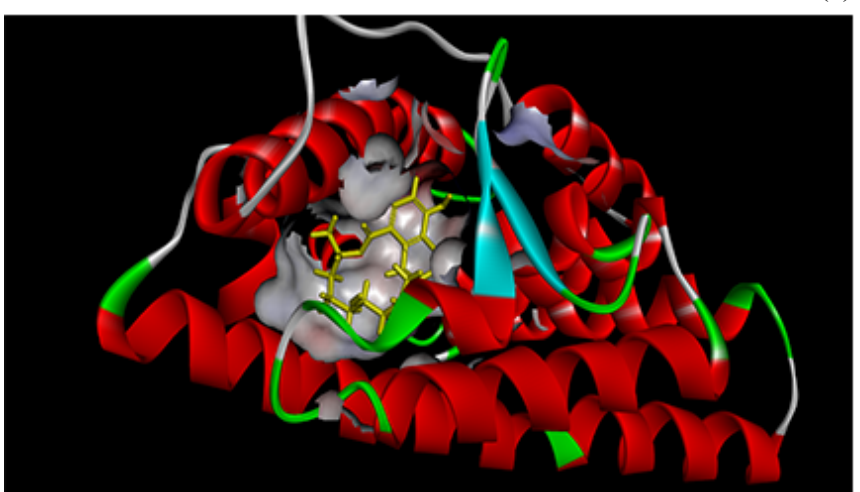

(b)
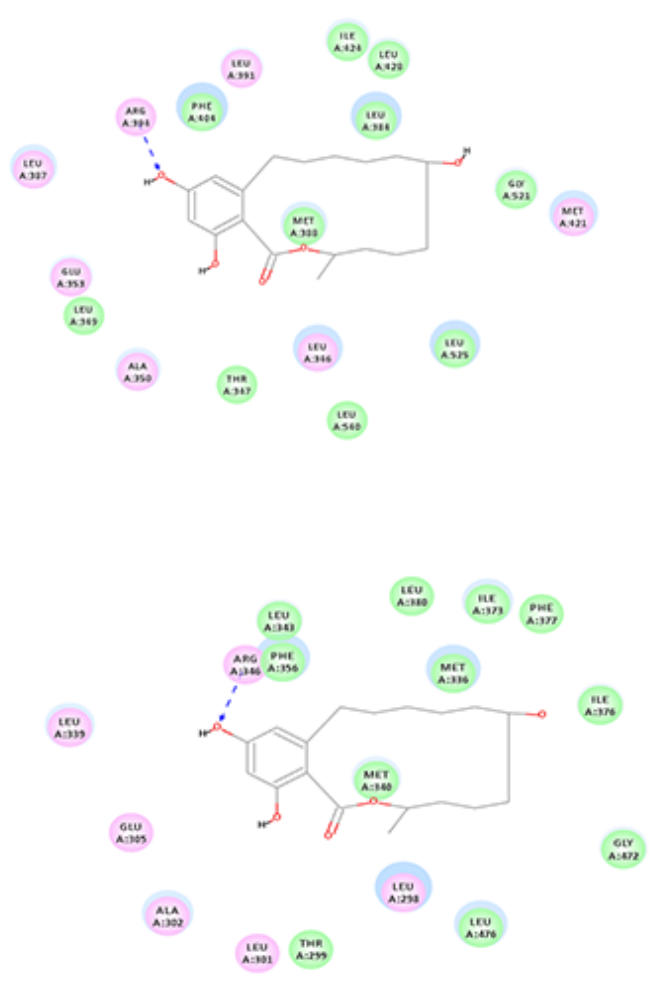

Fig. 12 The interaction between $\beta-Z O L$ and estrogen receptor protein.

Table 3 The binding energy and docking score between zearalenoneligands and receptors.

\begin{tabular}{llll}
\hline Ligand & Estrogen receptor protein & Binding energy $(\mathrm{kcal} / \mathrm{mol})$ & Libdock score \\
\hline $17 \beta$-estradiol & $\alpha$ - estrogen receptor protein & -26.8334 & 98.9602 \\
$17 \beta$-estradiol & $\beta$-estrogen receptor protein & -26.8334 & 108.235 \\
ZEN & $\alpha$ - estrogen receptor protein & -38.9894 & 113.57 \\
ZEN & $\beta$-estrogen receptor protein & -38.9894 & 106.956 \\
$\alpha$-ZOL & $\alpha$-estrogen receptor protein & -41.937 & 94.0647 \\
$\alpha$-ZOL & $\beta$-estrogen receptor protein & -41.937 & 72.6476 \\
$\beta$-ZOL & $\alpha$-estrogen receptor protein & -27.6144 & 115.28 \\
$\beta$-ZOL & $\beta$-estrogen receptor protein & -27.6144 & 107.458 \\
\hline
\end{tabular}

\section{Acknowledgement}

We are grateful to the State Key Laboratory of Biotherapy and Cancer Center, Collaborative Innovation Center for Biotherapy, West China Hospital, Sichuan University, Chengdu, China.

\section{References}

[1] Shoichet, B. K., Mcgovern, S. L., Wei, B., and Irwin, J. J. 2002. "Lead Discovery Using Molecular Docking."
Current Opinion in Chemical Biology 6: 439-46.

[2] Powers, C. N., and Setzer, W. N. 2015. “A Molecular Docking Study of Phytochemical Estrogen Mimics from Dietary Herbal Supplements." In Silico Pharmacology 3: $1-63$.

[3] Śledź, P., and Caflisch, A. 2017. "Protein Structure-Based Drug Design: From Docking to Molecular Dynamics." Curr Opin Struct Biol. 48: 93-102.

[4] Verdonk, M. L., Giangreco, I., Hall, R. J., Korb, O., Mortenson, P. N., and Murray, C. W. 2011. "Docking Performance of Fragments and Druglike Compounds." Journal of Medicinal Chemistry 54: 5422-31. 
[5] Leelananda, S. P., and Lindert, S. 2016. "Computational Methods in Drug Discovery." Beilstein Journal of Organic Chemistry 12: 2694-718.

[6] Macalino, S. J., Gosu, V., Hong, S., and Choi, S. 2015. "Role of Computer-Aided Drug Design in Modern Drug Discovery." Archives of Pharmacal Research 38: 1686-701.

[7] Eaton, D. L., and Gallagher, E. P. 1994. "Mechanisms of Aflatoxin Carcinogenesis." Annual Review of Pharmacoloy 34: 135-72.

[8] Koudande, D. O. 2013. “A Review on Aflatoxin Contamination and Its Implications in the Developing
World: A Sub-Saharan African Perspective.” Critical Reviews in Food Science and Nutrition 53: 349-65.

[9] Reed, K. F. M., Sprague, M., Mcfarlane, N. M., and Walsh, J. R. 2004. "Zearalenone and Its Presence in Pasture.” Animal Production in Australia 25: 140-3.

[10] Caldwell, R. W., Tuite, J., Stob, M., and Baldwin, R. 1970. "Zearalenone Production by Fusarium Species." Applied Microbiology 20: 31-4.

[11] Urry, W. H., Wehrmeister, H. L., Hodge, E. B., and Hidy, P. H. 1966. "The Structure of Zearalenone." Tetrahedron Letters 7: 3109-14. 